\title{
CEACAM6 acts as a receptor for adherent-invasive $E$. coli, supporting ileal mucosa colonization in Crohn disease
}

\author{
Nicolas Barnich,, ${ }^{1,2}$ Frédéric A. Carvalho,, 1,2 Anne-Lise Glasser, ${ }^{1,2}$ Claude Darcha, ${ }^{3}$ \\ Peter Jantscheff, ${ }^{4}$ Matthieu Allez, ${ }^{5}$ Harald Peeters, ${ }^{6}$ Gilles Bommelaer, ${ }^{7}$ \\ Pierre Desreumaux, ${ }^{8}$ Jean-Frédéric Colombel, ${ }^{8}$ and Arlette Darfeuille-Michaud ${ }^{1,2}$

\begin{abstract}
${ }^{1}$ Groupe de Recherche Pathogénie Bactérienne Intestinale, Université d’Auvergne, USC-INRA 2018, Clermont-Ferrand, France. 2Institut Universitaire de Technologie en Génie Biologique, Aubière, France. ${ }^{3}$ Service d'Anatomie et de Cytologie Pathologiques, CHU Hôtel-Dieu, Clermont-Ferrand, France. ${ }^{4}$ Tumor Biology Center, Freiburg, Germany. ${ }^{5}$ Service de Gastroentérologie, Hôpital Saint-Louis, Paris, France. ${ }^{6}$ Department of Gastroenterology, Ghent University Hospital, Ghent, Belgium. ${ }^{7}$ Service d'Hépato-Gastroentérologie, CHU Hôtel-Dieu,
\end{abstract} \\ Clermont-Ferrand, France. ${ }^{8}$ Physiopathologie des Maladies Inflammatoires Intestinales, INSERM U795, Lille, France.
}

\begin{abstract}
The ileal mucosa of Crohn disease (CD) patients is abnormally colonized by adherent-invasive E. coli (AIEC) that are able to adhere to and invade intestinal epithelial cells. Here, we show that CD-associated AIEC strains adhere to the brush border of primary ileal enterocytes isolated from $\mathrm{CD}$ patients but not controls without inflammatory bowel disease. AIEC adhesion is dependent on type 1 pili expression on the bacterial surface and on carcinoembryonic antigen-related cell adhesion molecule 6 (CEACAM6) expression on the apical surface of ileal epithelial cells. We report also that CEACAM6 acts as a receptor for AIEC adhesion and is abnormally expressed by ileal epithelial cells in CD patients. In addition, our in vitro studies show that there is increased CEACAM6 expression in cultured intestinal epithelial cells after IFN- $\gamma$ or TNF- $\alpha$ stimulation and after infection with AIEC bacteria, indicating that AIEC can promote its own colonization in CD patients.
\end{abstract}

\section{Introduction}

Idiopathic inflammatory bowel diseases (IBDs), which include Crohn disease (CD) and ulcerative colitis (UC), are chronic disorders of the gastrointestinal tract of unknown etiology with a combined prevalence of about $150-200$ cases per 100,000 in Western countries (1). The pathogenesis of $\mathrm{CD}$ is complex and consists of 3 interacting elements: genetic susceptibility factors, such as NOD2/CARD15; priming by the enteric microflora; and immune-mediated tissue injury (2-6). E. coli is the predominant aerobic Gram-negative species of the normal intestinal flora, where it plays an important role by promoting the stability of the luminal microbial flora and maintaining normal intestinal homeostasis. Modifications of luminal bacteria concentrations have been observed in $\mathrm{CD}$ patients. Indeed, the neoterminal ileum of $\mathrm{CD}$ patients is heavily colonized by a colonic-like bacterial flora, with $E$. coli predominating $(7,8)$. In a study to assess the predominance of $E$. coli strains associated with the ileal mucosa of CD patients, E. coli was recovered from $65 \%$ of chronic lesions (resected ileum) and from $100 \%$ of the biopsies of early lesions (postoperative endoscopic recurrence). E. coli were abnormally predominant (between $50 \%$ and $100 \%$ of the total number of aerobes and anaerobes) in early and chronic ileal lesions of CD patients (9). The presence of high concentrations of bacteria forming a biofilm on the surface of the gut mucosa was reported in patients with CD and UC (10). We observed that, in a given patient, healthy and ulcerated mucosa were colonized by E. coli strains hav-

Nonstandard abbreviations used: AIEC, adherent-invasive E. coli; $\mathrm{CD}$, Crohn disease; CEACAM, carcinoembryonic antigen-related cell adhesion molecule; ConA, concanavalin A; GPI, glycosylphosphatidylinositol; IBD, inflammatory bowel disease; LB, Luria-Bertani; THP, Tamm-Horsfall protein; UC, ulcerative colitis.

Conflict of interest: The authors have declared that no conflict of interest exists. Citation for this article: J. Clin. Invest. 117:1566-1574 (2007). doi:10.1172/JCI30504 ing the same ribotype profile, which indicates uniform colonization regardless of the inflammatory state of the mucosa (11). Thus, patients with $\mathrm{CD}$ appear to have defective handling of luminal bacteria and an increased number of mucosal bacteria (10).

The neoterminal ileum of $\mathrm{CD}$ patients is heavily colonized by E. coli strains able to adhere to and to invade cultured intestinal epithelial cells $(9,12)$. These E. coli strains, termed adherent-invasive E. coli (AIEC), are also able to survive extensively within macrophages and to induce the secretion of high amounts of TNF- $\alpha$ (13). Among the virulence factors harbored by AIEC strains, type 1 pili mediate adherence to intestinal epithelial cells and also play an essential part in the invasive ability of AIEC reference strain LF82 by inducing membrane extensions, which surround the bacteria at the sites of contact between the entering bacteria and the epithelial cells (14). In neoterminal ileal specimens, AIEC strains were found in $36.4 \%$ of early $\mathrm{CD}$ lesions and also in $22.2 \%$ of uninvolved mucosa of $\mathrm{CD}$ patients (15). However, AIEC strains do not represent a specific pathogen exclusively found in $\mathrm{CD}$ because their presence was also observed in $6.2 \%$ of ileal and $1.9 \%$ of colonic control specimens. The high prevalence of AIEC strains associated with the ileal mucosa observed in $\mathrm{CD}$ patients suggests an abnormal expression and/or tissue tropism of a specific host receptor recognized by bacterial lectin-like surface adhesins in a genetically predisposed host gut segment. Thus, we investigated the ability of CD-associated AIEC strains to colonize the ileal mucosa by analyzing their adhesion to isolated ileal enterocytes, the bacterial adherence factor involved, and the presence of an intestinal epithelial cell receptor responsible for bacterial adhesion.

\section{Results}

AIEC strain LF82 adheres to the brush border of primary ileal enterocytes from $C D$ patients. Adhesion of $C D$-associated AIEC reference strain LF82 was investigated using primary ileal epithelial cells isolated 
A

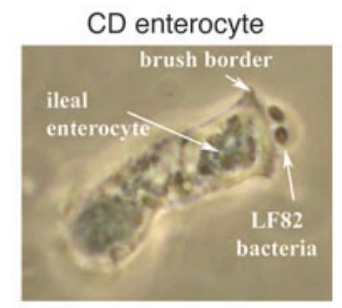

B

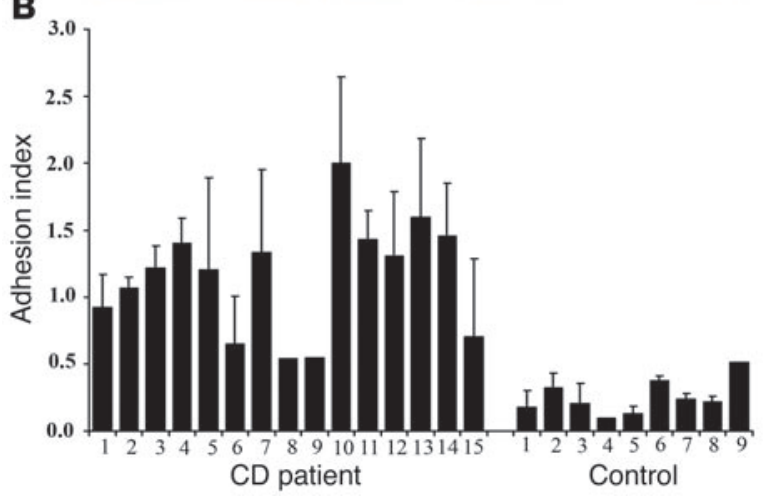

from $15 \mathrm{CD}$ patients and 9 controls without IBD. LF82 bacteria adhered preferentially to the brush border of ileal enterocytes isolated from CD patients (Figure 1A). Brush border adhesion indices of $E$. coli LF82, corresponding to the mean number of bacteria adherent to the brush border, ranged from 0.542 to 1.999 with enterocytes isolated from $\mathrm{CD}$ patients and from 0.096 to 0.511 with enterocytes from controls (Figure 1B). The ability of AIEC strain LF82 to adhere was therefore significantly higher with enterocytes isolated from $\mathrm{CD}$ patients than with those from controls $(P=0.0013)$. This supports the view that, in CD patients, a receptor involved in the adhesion of AIEC strain LF82 is expressed on the brush border of ileal enterocytes.

Adhesion of AIEC strains involves mannose residues and type 1 pili expression. As type 1 pili-mediated adherence to cultured intestinal epithelial cells plays an essential part in the virulence of AIEC strain LF82 (14), we investigated their involvement in the adhesion of LF82 bacteria to primary ileal enterocytes. In contrast to wild-type LF82, the LF82 type 1 pili-negative mutant 52D11 ( fim A::Tn5phoA) (Figure 2A) and the LF82 FimH adhesin-negative mutant ZG2 (fimH::Tn5phoA) (data not shown) did not adhere to the brush border of enterocytes isolated from $\mathrm{CD}$ patients. The adhesion levels of the wild-type strain LF82 on the brush border of primary enterocytes isolated from healthy donors were very low, and therefore we did not observe any significant decrease in the adhesion ability of the type 1 pili-negative mutants compared with that of the wild-type strain (Figure 2A). In addition, adhesion of LF82 strain to the brush border of CD enterocytes was blocked in the presence of $2 \%$ D-mannose (Figure 2B). However, when primary enterocytes isolated from healthy donors were used, D-mannose induced no significant decreases in the adhesion levels of LF82 bacteria on the brush border, mostly due to the very low adhesion levels observed even in the absence of $\mathrm{D}$-mannose (Figure 2B). This suggests that the interaction between AIEC LF82 type 1 pili and CD ileal enterocytes occurs via mannose residues expressed on the apical side of $\mathrm{CD}$ enterocytes. Adhesion experiments using primary ileal epithelial cells isolated from $\mathrm{CD}$ patients was extended to 4 other CD-associated AIEC strains

\section{Figure 1}

Adhesion of CD-associated AIEC strain LF82 to the brush border of ileal enterocytes. (A) Phase contrast microscopy of the adhesion of strain LF82 to the brush border of ileal enterocytes isolated from a CD patient and a control. Original magnification, $\times 1,000$. (B) Indices of adhesion of AIEC strain LF82 to the brush border of ileal enterocytes isolated from $15 \mathrm{CD}$ patients and 9 controls. The number of bacteria adherent to the brush border of 30-50 enterocytes was determined. Adhesion index was expressed as the mean number of bacteria adherent to the brush border of 1 enterocyte. Two separate experiments were performed in duplicate.

(LF31, LF71, LF73, and LF100). As shown in Table 1, all AIEC strains tested, like strain LF82, were able to adhere to the brush border of primary enterocytes isolated from CD patients. Their ability to adhere was blocked using $2 \%$ D-mannose (Table 1 ), and corresponding type 1 pili-negative mutants were unable to adhere to the brush border (Table 1). These results demonstrate that the ability of bacteria to adhere via a type 1 pili-mediated adherence pathway to the brush border of primary ileal enterocytes from $C D$ patients is not restricted to the AIEC reference strain LF82 but is a characteristic of CD-associated AIEC strains.

AIEC strains expressed type 1 pili variants. The adhesion level of AIEC LF82 expressing such type 1 pili variant was substantially higher than that of a nonpathogenic E. coli K-12 in which an overexpression of regular type 1 pili was induced (Figure 2C). This was not due to differences in type 1 pili expression, since Western blot analysis revealed similar amounts of type 1 pili expression in these 2 strains (Figure 2C). To confirm the greater ability of LF82 type 1 pili variant compared with the K-12 type 1 pili to adhere, we engineered an AIEC LF82 strain expressing E. coli K-12 type 1 pili. AIEC strain LF82 expressing K-12 type 1 pili was able to adhere, but to a lesser extent than AIEC strain LF82 expressing type 1 pili variant (Figure 2C). To further analyze the adhesion of AIEC strain LF82 to the brush border of ileal enterocytes involving type 1 pili expression, enterocytes from $\mathrm{CD}$ patients or controls were incubated with FITC-labeled concanavalin A (ConA), which binds to mannose. High levels of ConA bound to the brush border of enterocytes from $\mathrm{CD}$ patients but not to that of enterocytes from controls (Figure 2D). These results demonstrate a high expression of mannosylated molecule(s) on the apical surface of the primary ileal enterocytes of $\mathrm{CD}$ patients.

Expression of carcinoembryonic antigen-related cell adhesion molecules 5 and 6 is upregulated in $C D$ ileal biopsies. Several glycosylated receptors for type 1 pili have been documented, such as glycosylphosphatidylinositol-anchored (GPI-anchored) protein CD48, Tamm-Horsfall protein (THP), and various family members of carcinoembryonic antigen-related cell adhesion molecules (CEACAMs) (16-20). Western blot analysis of total protein extract from ileal specimens of $9 \mathrm{CD}$ patients, taken in both involved and uninvolved areas, and of 9 controls showed a strong expression of CEACAM5 (also called CEA or CD66e) and CEACAM6 (also called CD66c or non-crossreacting antigen $[\mathrm{NCA}]$ ) in the ileal mucosa of $\mathrm{CD}$ patients in both involved and uninvolved areas (Figure 3A). No expression of THP, CD48, or CEACAM1 was found (data not shown). Blots of total cell lysates were probed with ConA-HRP to determine how many glycoproteins were upregulated or had increased mannose. The overall intensity of the signal was higher in ileal CD specimens compared with controls (data not shown). However, it was not possible to determine the number of glycoproteins upregulated in 

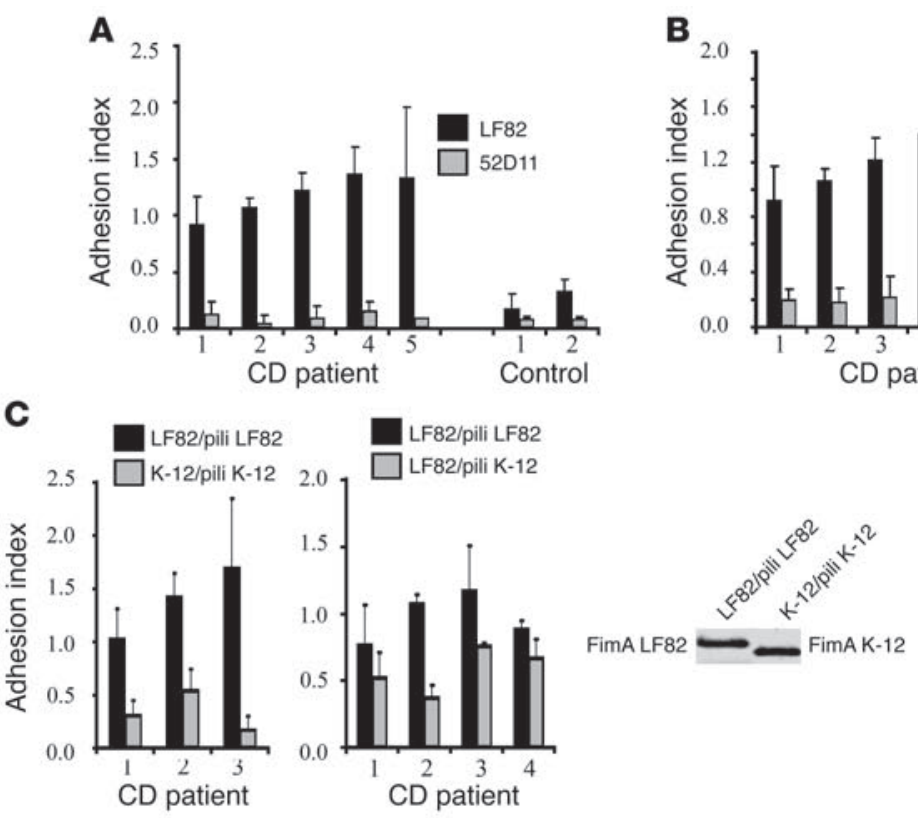

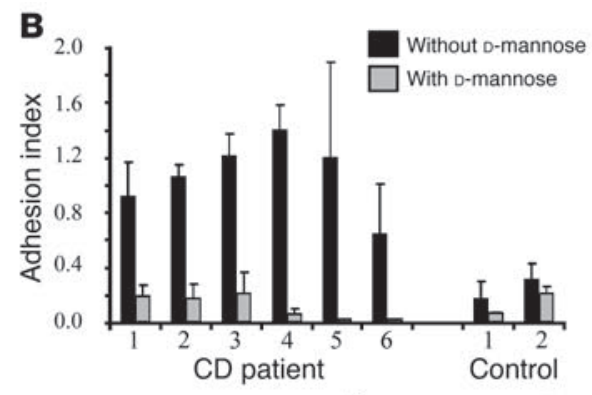

D
Fluorescence labeling (ConA-FITC)

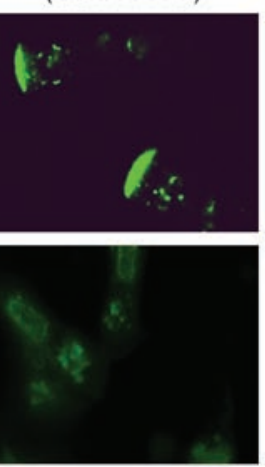

\section{Figure 2}

Type 1 pili-mediated adhesion to the brush border of ileal enterocytes. (A) Adhesion of the LF82-derived type 1 pili-negative mutant 52D11 (fimA:: Tn5phoA) to the brush border of enterocytes isolated from CD patients and controls. (B) Adhesion of AIEC LF82 to the brush border of enterocytes isolated from CD patients and controls in the absence or presence of $2 \% \mathrm{D}$-mannose. (C) Adhesion of LF82 bacteria expressing type 1 pili variant and of nonpathogenic $E$. coli K-12 transformed with pPil38 to induce the expression of K-12 type 1 pili (left panel) or of LF82 bacteria expressing either LF82 type 1 pili variant or K-12 type 1 pili (middle panel) to the brush border of enterocytes isolated from CD patients. Western blot analysis using anti-type 1 pili antibodies performed on whole bacterial extracts showed similar amounts of type 1 pili expressed by the various strains (right panel). (D) Expression of a highly mannosylated molecule on the brush border of ileal enterocytes isolated from CD patients. Phase contrast microscopy and fluorescence labeling using ConA-FITC of ileal enterocytes from a CD patient and a control. Original magnification, $\times 400$.

CD ileal specimens. A basal expression of CEACAM6 was observed in the colonic mucosa of the 3 control patients without IBD tested (Figure 3B). No upregulation of CEACAM6 expression was observed in the colonic mucosa, in contrast to the ileal mucosa, of CD patients. We also observed that CEACAM6 expression was not elevated in the colon of UC patients (Figure 3B). These results indicate that the upregulation of CEACAM6 expression is specific to the ileal segment of the intestine of $C D$ patients.

AIEC LF82 bacteria recognize CEACAM6 receptor on CD ileal enterocytes. Pretreatment of primary ileal enterocytes isolated from CD patients with an anti-CEACAM6 monoclonal antibody strongly decreased LF82 adhesion, whereas pretreatment with anti-CD48, -CEACAM1, -CEACAM5, or -THP monoclonal antibodies had no effect on LF82 adhesion (Figure 3C). LF82 adhesion inhibition using anti-CEACAM6 antibodies was observed with all 4 specimens from individual CD patients (Figure 3D). Confocal microscopy examination of HeLa cells transfected with cloned human CEACAM6 cDNA showed that AIEC LF82 bacteria adhered preferentially to HeLa cells expressing a high level of CEACAM6 (Figure 3E). This adhesion could be blocked in the presence of D-mannose, and no adhesion was observed with the nonpiliated LF82 mutants (data not shown). Taken together, these data clearly demonstrate that adhesion of E. coli strain LF82 to the ileal mucosa of CD patients involves type 1 pili binding to the GPI-anchored receptor CEACAM6 expressed on the apical side of ileal epithelial cells.
CD patients express a high level of CEACAM6 molecules on the ileal epithelium. Immunohistochemical analysis was performed on the ileal and colonic mucosa of $20 \mathrm{CD}$ patients and 20 controls using mouse anti-human CEACAM6 monoclonal antibody (clone 9A6). In $C D$ patients, positive staining was observed on both uninvolved and involved ileal mucosa in 11 individuals and on inflamed mucosa alone in 6 others. In uninvolved mucosa of CD patients, a focal expression of CEACAM6 was observed on the apical surface of ileal epithelial cells with a marked gradient between villus and crypt epithelial cells (Figure 4A, top left). Examination of inflamed areas of ileal mucosa from patients expressing CEACAM6 on uninvolved mucosa revealed a stronger expression of CEACAM6 on the apical surface and the cytoplasm of epithelial cells, as well as on the surface of goblet cells and infiltrating immune cells known to express CEACAM6 (21) (Figure 4A, middle left). In 2 patients undergoing surgery for ileal stenosis, we observed strong staining of the ileal epithelial cells together with staining of infiltrating immune cells (Figure 4A, bottom left). No staining was observed with the isotype-matched $\left(\operatorname{IgG}_{1}\right)$ control (Figure $4 \mathrm{~A}$, right column). In 17 of the 20 controls tested, no staining of ileal epithelial cells was observed (Figure 4B, left), even in areas where infiltrating immune cells were present (Figure 4B, right). A focal expression of CEACAM6 similar to that shown in Figure 4A (top left) was observed with ileal specimens of 3 controls. Two had right colon cancer, and 1 had ileal ischemia of unknown etiology. 


\section{Table 1}

Ability of various AIEC strains and the corresponding fimH mutants to adhere to the brush border of primary ileal enterocytes isolated from CD patients

\begin{tabular}{|c|c|c|c|c|c|c|}
\hline \multirow{3}{*}{$\begin{array}{l}\text { CD patient } \\
1\end{array}$} & \multicolumn{6}{|l|}{ AIEC strain } \\
\hline & Group & LF82 & LF31 & LF71 & LF73 & LF100 \\
\hline & WT & $0.93 \pm 0.24$ & $1.08 \pm 0.31$ & $0.83 \pm 0.03$ & $0.88 \pm 0.39$ & $0.86 \pm 0.38$ \\
\hline & $\Delta$ fimH mutant & $0.13 \pm 0.11^{A}$ & $0.07 \pm 0.04^{A}$ & $0.17 \pm 0.03^{A}$ & $0.05 \pm 0.07^{A}$ & $0.03 \pm 0.03^{A}$ \\
\hline & + D-mannose ${ }^{B}$ & $0.20 \pm 0.07^{A}$ & $0.11 \pm 0.03^{\mathrm{A}}$ & $0.03 \pm 0.04^{A}$ & $0.19 \pm 0.06^{A}$ & $0.10 \pm 0.05$ \\
\hline 2 & WT & $1.07 \pm 0.09$ & $1.37 \pm 0.52$ & $0.75 \pm 0.35$ & $0.55 \pm 0.14$ & $0.83 \pm 0.21$ \\
\hline & $\Delta$ fimH mutant & $0.05 \pm 0.07^{A}$ & $0.07 \pm 0.10^{\mathrm{A}}$ & $0.13 \pm 0.03^{A}$ & $0.35 \pm 0.05$ & $0.08 \pm 0.04$ \\
\hline & + D-mannose ${ }^{B}$ & $0.18 \pm 0.11^{A}$ & $0.30 \pm 0.11^{\mathrm{A}}$ & ND & ND & $0.25 \pm 0.07$ \\
\hline 3 & WT & $1.22 \pm 0.16$ & $1.26 \pm 0.30$ & $0.77 \pm 0.07$ & $1.15 \pm 0.38$ & $1.01 \pm 0.29$ \\
\hline & $\Delta$ fimH mutant & $0.10 \pm 0.10^{A}$ & $0.11 \pm 0.07^{\mathrm{A}}$ & $0.08 \pm 0.04^{A}$ & $0.35 \pm 0.27$ & $0.18 \pm 0.16^{f}$ \\
\hline & + D-mannose ${ }^{B}$ & $0.22 \pm 0.15^{A}$ & $0.25 \pm 0.10^{\mathrm{A}}$ & $0.23 \pm 0.11$ & $0.25 \pm 0.14$ & $0.28 \pm 0.15$ \\
\hline
\end{tabular}

Values represent adhesion index. ${ }^{A} P<0.05 .{ }^{B}$ Adhesion indices of wild-type strains in the presence of $2 \% \mathrm{D}$-mannose. ND, not determined.

Collectively, immunostaining examination revealed significantly increased expression of CEACAM6 in the uninvolved or inflamed ileal mucosa of CD patients compared with the mucosa of controls without IBD. The expression of CEACAM6 on the surface of ileal epithelium was absent in $85 \%$ of the controls without IBD, moderate in $50 \%$ of CD patients and $15 \%$ of controls, and strong to very strong in $35 \%$ of $C D$ patients (Figure $4 C$ ). Statistical analysis indicated that the prevalence of strong to very strong ileal expression of CEACAM6 was higher in patients with CD than in controls $(P=0.0007)$. Interestingly, significantly higher expression of CEACAM6 was also found in uninvolved ileal mucosa of CD patients compared with that of controls $(P=0.008)$. Analysis of colonic specimens showed that CEACAM6 was expressed on colonic mucosa of all CD patients and controls. There was no marked difference in CEACAM6 expression between CD patients and controls except in those with colorectal cancer (data not shown).

CEACAM6 expression is upregulated by proinflammatory cytokines and AIEC infection in intestinal epithelial cells. Of the various intestinal epithelial cell lines used in this study, only Caco-2, LS174, and T84 expressed CEACAM6 (Figure 5A). However, no correlation was observed between the adhesion levels of AIEC strain LF82 and the levels of CEACAM6 expression with the various cell lines tested (data not shown). The reason may be that the repertoire of glycoproteins abnormally expressed or abnormally glycosylated in all these undifferentiated cancer cell lines is very broad. When differentiated Caco-2 cells infected with strain LF82 bacteria were used, a clear colocalization between CEACAM6 expression and adherent bacteria was observed. This was confirmed by $3 \mathrm{D}$ reconstruction images showing apical expression of CEACAM6 beneath adherent bacteria (Figure 5B).

IFN- $\gamma$ or TNF- $\alpha$ stimulations, but not LF82 infection, weakly induced CEACAM5 in Caco- 2 cells, whereas no CEACAM1 expression was observed (Figure 5C). IFN- $\gamma$ strongly increased CEACAM6 protein expression and, to a lesser extent, TNF- $\alpha$ also increased CEACAM6 expression. Interestingly, an increase in CEACAM6 protein level was observed in Caco- 2 cells after 3 hours of infection by AIEC strain LF82 at an MOI of 10 (Figure 5C). In addition, neither LF82 infection nor IFN- $\gamma$ or TNF- $\alpha$ stimulation induced CEACAM6 expression on Colo205, SW480, and HCT-116 intestinal epithelial cells in which no endogenous CEACAM6 protein was detected (data not shown). This indicates that inflamma- tory conditions or infection with pathogenic bacteria can induce CEACAM6 expression only in intestinal epithelial cells expressing CEACAM6 at a basal level. Finally, the ability of AIEC strain LF82 to adhere to Caco- 2 cells was significantly increased in a dose-dependent manner when the expression of CEACAM 6 was induced after 1 and 2 days of IFN- $\gamma$ stimulation (Figure 5D). In addition, this effect was reversed in the presence of CEACAM6 siRNA, whereas control siRNA, which did not reduce CEACAM6 protein levels, had no effect on AIEC LF82 adhesion (Figure 5D). Together, these findings demonstrate that the AIEC adhesion level increases with CEACAM6 expression by intestinal epithelial cells.

\section{Discussion}

We show that CD-associated AIEC strains adhere to the brush border of primary ileal enterocytes prepared from CD patients but not to enterocytes from controls without IBD. In normal human gut, commensal E. coli are not able to adhere to the apical face of intestinal epithelial cells. Only pathogenic E. coli, which have acquired, by horizontal transfer, virulence genes encoding adhesive factors have the ability to adhere to the apical surface of differentiated intestinal epithelial cells. For example, enterotoxigenic E. coli (ETEC) synthesize specific adhesive factors called colonization factor antigens (CFAs) (22) that specifically bind asialoganglioside GM1 expressed on the brush border of small intestine enterocytes $(23,24)$. In a more specific manner, enteropathogenic E. coli (EPEC) or enterohemorrhagic E. coli (EHEC) use a type III secretion machinery to attach to intestinal epithelial cells, translocating their own receptor for intimate attachment (translocated intimin receptor [Tir]) into the host cell, which then binds to intimin on the bacterial surface $(25,26)$. Finally, Afa/Dr adhesins of diffusely adhering E. coli (DAEC) allow bacteria to bind to human decay-accelerating factor (DAF) or carcinoembryonic antigen molecules (27). We previously reported that AIEC strains do not possess any of the genes encoding known adhesive factors of pathogenic E. coli strains involved in either intestinal or extraintestinal infections but do express type 1 pili $(9,14)$. Type 1 pili are common filamentous bacterial appendages, and the FimH adhesin located at the tip of the fibrillum structure can mediate bacterial adhesion to nonglycosylated host receptors, including the matrix-associated type I and IV collagens, laminin and fibronectin, and to glycosylated receptors (28). Most AIEC strains asso- 
A

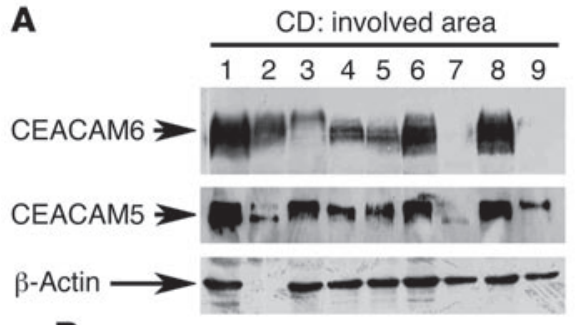

B

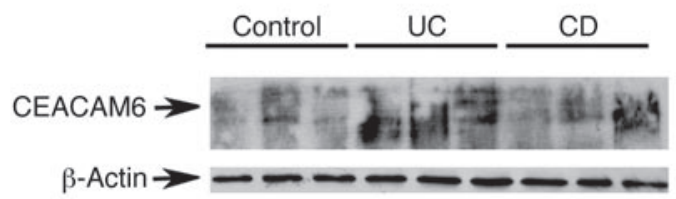

D

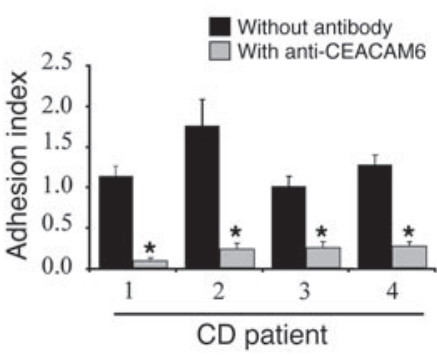

CD: noninvolved area

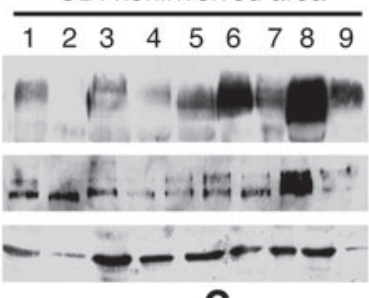

C

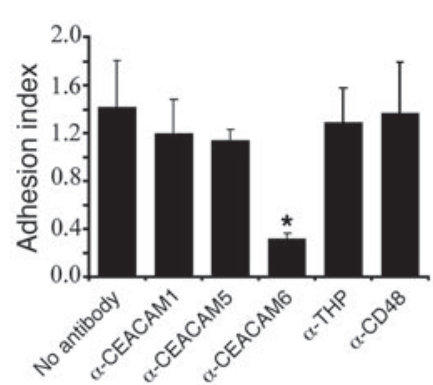

E

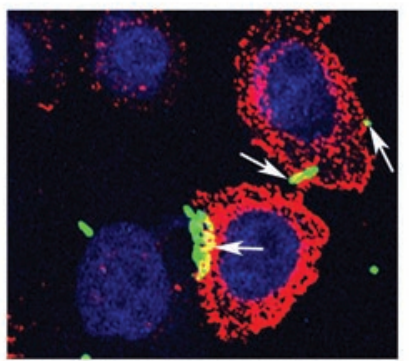

Figure 3

CEACAM6 expression supports AIEC adhesion. (A) Western blot analysis of whole protein extracts from ileal biopsies taken in both involved and uninvolved areas of $9 \mathrm{CD}$ patients and from 9 ileal biopsies from controls using antiCEACAM5, anti-CEACAM6, and anti- $\beta$-actin antibodies. (B) Western blot analysis of whole protein extracts from colonic biopsies from 3 controls, 3 UC patients, and 3 CD patients using anti-CEACAM 6 and anti- $\beta$-actin antibodies. (C) Adhesion indices of AIEC strain LF82 to the brush border of enterocytes from CD patients in the presence of monoclonal antibodies raised against CEACAM1, CEACAM5, CEACAM6, THP, and CD48. (D) Adhesion indices of AIEC strain LF82 to the brush border of enterocytes from 4 CD patients in the presence of anti-CEACAM6 monoclonal antibody. (E) Confocal microscopic analysis of HeLa cells transfected with human CEACAM6 cDNA cloned and infected with AIEC LF82 harboring a GFP construct. Original magnification, $\times 1,000$. CEACAM6 expression was detected using anti-CEACAM6 monoclonal antibody and a Texas red-conjugated anti-mouse IgG. Arrows show clear colocalization (yellow staining) between CEACAM6 and bacteria.

ciated with CD ileal mucosa express a type 1 pili variant (14), and interestingly we observed in the present study that expression of type 1 pili variant, compared with that of K-12 type 1 pili, enhances bacterial adhesion to the ileal enterocyte brush border. This could be a possible explanation of the high prevalence of AIEC associated with the ileal mucosa in CD patients (15).

AIEC adhesion to ileal enterocytes of CD patients was blocked in the presence of D-mannose, indicating that bacteria-host cell interactions occur via glycosylated receptors. In addition, an elevated expression of mannosylated molecule(s) on ileal enterocytes of $\mathrm{CD}$ patients was observed using the mannose-binding lectin ConA. This could indicate the presence of: (a) a receptor differentially expressed between CD patients and controls; or (b) truncated forms of oligosaccharide chains with mannose residues present in the terminal position owing to incomplete glycosylation. The latter hypothesis has been suggested by results in patients with UC (29-31). As we found that the adhesion of AIEC strains occurs via type 1 pili, we focused on the expression of known type 1 pili
Previous reports have evidenced a selectively enhanced expression of CEACAM6 but not of CEACAM5 in the serum of CD patients, indicating a stronger upregulation of the former molecule (32). These findings suggest that the high level of CEACAM6 observed in $\mathrm{CD}$ patient sera could result from the overexpression of CEACAM6 by ileal epithelial cells. CEACAM6 is expressed by colonic epithelial cells and is upregulated in colorectal tumors (32-34), but we report here that CEACAM6 is not upregulated in the colonic mucosa of IBD patients. However, there is documented evidence that CEACAM6 is not expressed by epithelial cells of the small intestine in non-IBD patients (35) and that normal mucosa does not generally display ConA binding activity (36).

Overexpression of CEACAM6 by ileal epithelial cells in CD patients can be induced in at least 2 different ways, either by direct stimulation with pathogenic bacteria or by stimulation with proinflammatory cytokines. The in vitro experiments reported here show that CEACAM6 expression in cultured intestinal epithelial cells is upregulated both by AIEC infection and by stimulation with 
A $C D$ patients
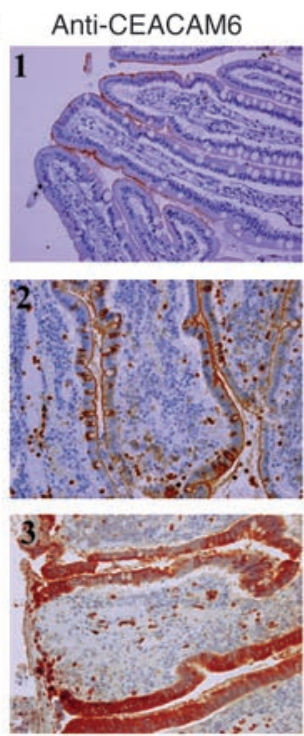

Isotype control
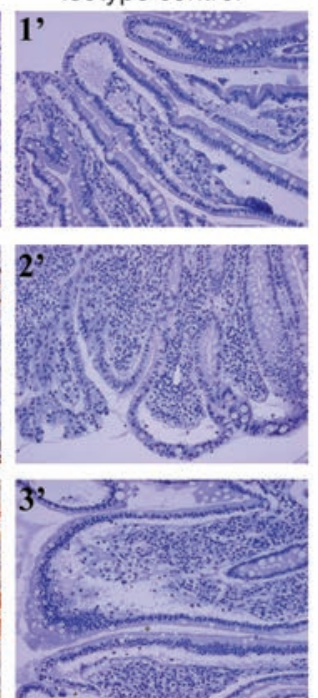

B Control patients
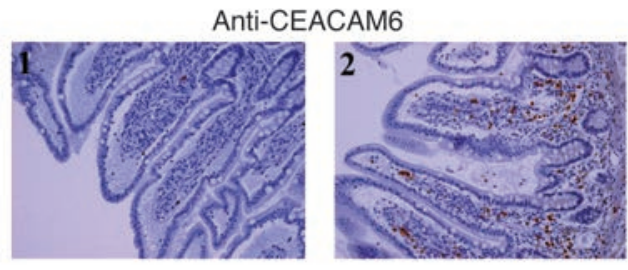

C

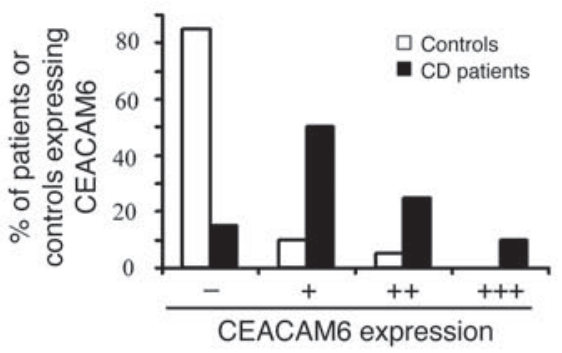

Figure 4

Tissue expression of CEACAM6. Immunohistochemistry staining of ileal sections from CD patients (A) and controls (B) using anti-CEACAM6 monoclonal antibody clone 9A6 or isotype control. Original magnification, $\times 100$. (C) Analysis of CEACAM6 expression in 20 CD patients and 20 controls monitored from negative $(-)$ to strongly positive $(+++)$ staining as described in Methods.

the proinflammatory cytokines IFN- $\gamma$ and TNF- $\alpha$. Upregulation of CEACAM molecules by IFN- $\gamma$ stimulation has been previously documented (37). The fact that TNF- $\alpha$ is also able to induce increased expression of CEACAM6 is of interest, since macrophages infected with AIEC bacteria secrete large amounts of TNF- $\alpha$ (13). Thus, AIEC bacteria can promote their own colonization in CD patients directly by inducing an increased expression of CEACAM6 by intestinal epithelial cells and indirectly via the induced secretion of TNF- $\alpha$ by infected macrophages. We believe this to be a novel example of a bacterial pathogen subverting functional membranebound proteins as receptors for colonizing epithelia and exploiting cell signaling pathways to cross-talk with the host cells. Likewise, Neisseria gonorrboeae upregulates the opacity-associated (Opa) protein adhesion receptor CEACAM1 on primary endothelial cells (38); Afa/Dr DAEC infection induces increased neutrophil transepithelial migration, which in turn promotes cytokine-dependent upregulation of the Afa/Dr receptor CD55 (39); and adhesion of nontypeable Haemophilus influenzae upregulates the expression of its receptor, ICAM-1, by respiratory epithelial cells (40).

The issue of whether the upregulation of CEACAM6 expression in the ileal mucosa of $\mathrm{CD}$ patients is a primary or secondary consequence of CD remains unclear. A basal expression of CEACAM6 was observed in macroscopically uninvolved ileum in the majority of CD patients, which suggests that CEACAM6 expression is not merely a consequence of active inflammation. However, it could be due to sustained immune stimulation with increased levels of inflammatory cytokines even in the absence of patent inflammation, as already reported for the intestinal mucosa of CD patients (41). Alternatively, inducible expression of CEACAM6 in the ileal mucosa of CD patients could be genetically linked. We observed that cytokine stimulation of intestinal epithelial cells that did not express CEACAM6 did not induce CEACAM6 expression. The significantly increased ileal CEACAM6 expression found in uninvolved ileal mucosa of CD patients compared with controls without IBD could suggest that patients expressing a basal level of CEACAM6 are genetically predisposed to overexpression of that molecule. Of note, the gene encoding CEACAM6 is located on the long arm of human chromosome 19 (q13.2 between CYP2A and D19S15), close to a suggested IBD susceptibility locus (42).

Studies on the prevalence of AIEC strains in IBD patients and controls indicated that these pathogenic strains are isolated mainly from patients with CD and associated with ileal mucosa (36.4\%) but rarely found in colonic mucosa (3.7\%). AIEC strains are rarely associated with ileal or colonic mucosa of controls, or with colonic mucosa of UC patients (15). We observed a basal expression of CEACAM6 in the colonic mucosa of CD and UC patients and controls, which was consistent with findings of a previous report (35). Thus, a higher prevalence of AIEC in the colonic mucosa could have been expected. One possible explanation is that the concentration of bacteria is higher in the colon than in the ileum, and therefore AIEC bacteria can be in competition with the normal flora to gain access to the CEACAM6 receptor.

In conclusion, our findings show that CEACAM6 acts as a host cell receptor for AIEC adhesion via a type 1 pili variant and suggest a causal role of CEACAM6 expression in colonization of the ileal mucosa of CD patients by AIEC strains. However, it remains to be determined whether CEACAM6 overexpression is due to the presence of AIEC or AIEC colonization is a consequence of CEACAM6 upregulation. Hence, in addition to NOD2/CARD15, abnormal ileal CEACAM6 expression could be another susceptibility factor for CD. Accordingly, patients expressing a basal level of CEACAM6 would be genetically predisposed to develop ileal CD, and the presence of AIEC bacteria and the secretion of IFN- $\gamma$ and TNF- $\alpha$ would lead to an amplification loop of colonization and inflammation. We speculate that patients at high risk for developing severe ileal $\mathrm{CD}$ are those who, in addition to expressing a variant of the NOD2 intracytoplasmic receptor $(5,6)$, overexpress CEACAM6 in the ileal mucosa. The new light thrown upon the etiology of ileal CD by our 


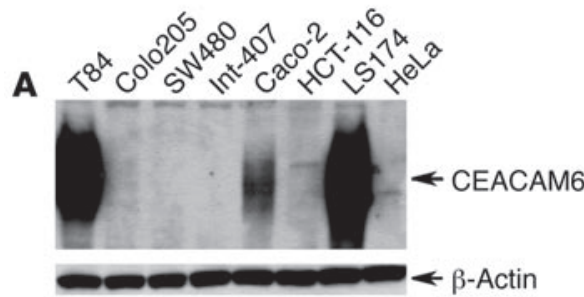

B
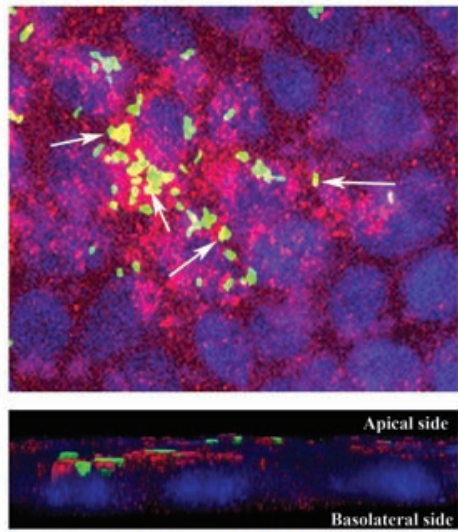

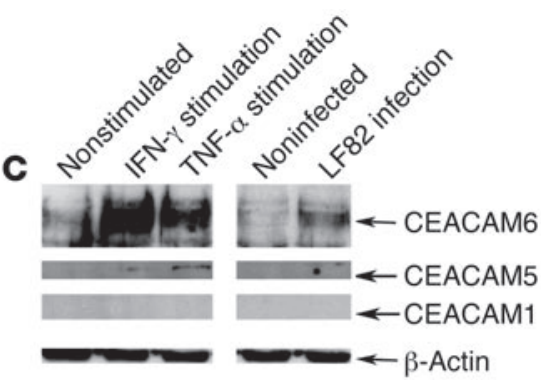

D

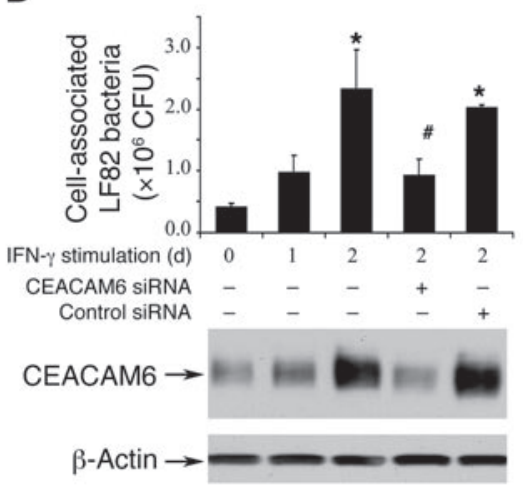

Figure 5

CEACAM6 expression and LF82 adhesion ability with various intestinal epithelial cells. (A) Western blot analysis using monoclonal antibodies CEACAM 6 clone $9 A 6$ and anti- $\beta$-actin. Ten micrograms of total protein from different intestinal epithelial cell lines were loaded onto $4 \%-12 \%$ Tris-glycine gel. (B) Confocal microscopic analysis of differentiated Caco-2 cells infected with GFP-expressing LF82 bacteria. Original magnification, $\times 400$. CEACAM6 was detected using anti-CEACAM6 monoclonal antibody clone 9A6 and a Texas red-conjugated anti-mouse IgG (top panel). Arrows show colocalization (yellow) between CEACAM6 and bacteria. A 3D reconstruction (bottom panel) showed apical expression of CEACAM6 (red) and adherent bacteria (green). (C) Western blot analysis showing expression levels of CEACAM6, CEACAM5, and CEACAM1 by Caco-2 cells after 48 hours of stimulation with IFN- $\gamma$ or TNF- $\alpha$ or after a 3-hour infection period with AIEC LF82 bacteria at an MOI of 10. As loading control, a labeling was performed using anti- $\beta$-actin polyclonal antibodies (D) Adhesion ability of AIEC LF82 bacteria was quantified after a 3-hour infection period at an $\mathrm{MOI}$ of 10 in Caco-2 intestinal epithelial cells after 1 and 2 days of IFN- $\gamma$ stimulation. For RNA silencing, IFN- $\gamma-$ stimulated Caco-2 cells were transfected with $10 \mathrm{ng}$ of siRNA-blocking CEACAM6 (CEACAM6 siRNA) or $10 \mathrm{ng}$ of nonworking siRNA (control siRNA). Expression of CEACAM6 was analyzed by Western blot analysis using anti-CEACAM6 monoclonal antibody clone 9 A6 or anti- $\beta$-actin polyclonal antibodies. ${ }^{*} P<0.05$ compared with nonstimulated Caco-2 cells; $\# P<0.05$ compared with Caco-2 cells stimulated for 2 days with IFN- $\gamma$ and untransfected or transfected with control siRNA.

findings suggests that ileal CEACAM6 expression in IBD patients could be a diagnostic marker for ileal CD.

\section{Methods}

Patients. A total of 35 patients with ileal or ileocolonic CD and 29 controls without IBD were included in this study. All patients and controls gave informed consent, and approval from Comité Consultatifs de Protection des Personnes dans la Recherche Biomédicale ([CCPPRB], Lille, France) was obtained. The diagnosis of $\mathrm{CD}$ was established using previously published criteria (43). The preparation of isolated ileal enterocytes was performed with resection specimens from 15 patients with $C D$ ( 7 women, 8 men; mean age, 30 years; range, $17-52$ years). Seven patients had undergone surgery for ileal involvement of $\mathrm{CD}$ and 8 for ileocolonic $\mathrm{CD}$. Enterocytes were also prepared from ileal surgery specimens or endoscopic biopsies from 9 controls without IBD ( 5 women, 4 men; mean age, 46 years; range, 16-70 years), of whom 3 had undergone right hemicolectomy for cancer.

The immunohistochemistry assays for CEACAM6 staining were performed on paraffin-embedded ileal and colonic biopsies from $20 \mathrm{CD}$ patients (14 women, 6 men; mean age, 38 years; range, $19-69$ years). Eleven patients had only ileal involvement of CD, and 9 had ileocolonic CD. Analysis was also performed on ileal biopsies from 20 controls without IBD (14 women, 6 men; mean age, 58 years; range, 1 month-93 years), of whom 3 had indeterminate colitis with ileal inflammation and 4 had undergone right hemicolectomy for colonic cancer.

Western blot analysis of CEACAM6 expression by colonic specimens was performed on colonic biopsies from $3 \mathrm{CD}$ patients ( 2 women, 1 man; mean age, 24 years; range, 20-26 years), 3 UC patients ( 1 woman, 2 men; mean age, 47 years; range, $34-54$ years), and 3 controls ( 2 women, 1 man; mean age, 52 years; range, 37-62 years).

Bacterial strains, plasmids, and bacterial culture. E. coli strain LF82, isolated from a chronic ileal lesion of a CD patient, was used as the reference strain for AIEC, as well as 4 other AIEC strains, LF31, LF71, LF73, and LF100 (12). LF82 type 1 pili-negative mutants harboring an insertion of the transposon Tn5phoA into fimA (52D11) or fimH (ZG2) gene were previously generated (14). AIEC- $\Delta$ fim $H$ isogenic mutants were generated with a PCR product, using the method described by Datsenko and Wanner (44) and modified by Chaveroche et al. (45). The recombinant $E$. coli K-12 strain harboring pPil38 containing the entire cloned fim operon was used for adhesion assays (46). Bacteria were grown routinely in Luria-Bertani (LB) broth or on LB or Mueller-Hinton agar plates (BD) overnight at $37^{\circ} \mathrm{C}$ without shaking.

In vitro adhesion to isolated enterocytes. In vitro adhesion assays were performed as previously described $(22,24)$. Isolated enterocytes were prepared from ileal specimens frozen at $-80^{\circ} \mathrm{C}$ in MEM (Seromed Biochrom) containing $10 \%$ glycerol and $10 \%$ DMSO (Sigma-Aldrich) immediately after removal. Frozen intestinal samples were washed 3 times in PBS ( $\mathrm{pH}$ 7.2), and the ileal mucosa was scraped with a coverslip to detach enterocytes. Approximately $10^{5}$ isolated enterocytes were mixed with $10^{8}$ E. coli cells in MEM containing $10 \%$ of heat-inactivated FCS (Seromed Biochrom). After a 2-hour incubation period at $37^{\circ} \mathrm{C}$ with gentle shaking, enterocytes were washed 3 times in PBS. Bacterial adhesion was quantified by examination under phase contrast microscopy at a magnification of $\times 1,000$. The number of $E$. coli bacteria adhering to the brush border of 30-50 enterocytes was counted in duplicate. Experiments with enterocytes were performed in duplicate by at least 2 different experimenters. The adhesion index was expressed as the mean number of bacteria attached to the brush border of 1 enterocyte. 
Inhibition adhesion assays were performed either in the presence of $2 \%$ (wt/vol) D-mannose (Sigma-Aldrich) in MEM containing 10\% FCS or after a 30 -minute pretreatment of enterocytes at $37^{\circ} \mathrm{C}$ using various antibodies. Bacteria were then added, and adhesion assays were performed as described above. Monoclonal anti-CD48, anti-CEACAM1, anti-CEACAM5, and antiTHP (PeliCluster; Tebu-Bio) and monoclonal anti-CEACAM6 clone 9A6 antibodies were used at 1:100 dilution in MEM containing 10\% FCS.

Analysis of the presence of free mannose residues on enterocyte brush border. Isolated enterocytes were incubated for 1 hour in PBS containing $50 \mu \mathrm{g} / \mathrm{ml}$ of ConA-FITC (Sigma-Aldrich) lectin, which specifically binds to mannose residues. After 4 washes, enterocytes were observed by fluorescence microscopy at a magnification of $\times 400$.

Immunohistochemistry on ileal specimens. Immunohistochemistry was performed on 4- $\mu \mathrm{m}$ sections of formalin-fixed paraffin-embedded tissue samples using the avidin-biotin peroxidase complex method and 3-amino,9ethyl-carbazole chromogen. Sections were subjected to heat-induced epitope retrieval using a pressure cooker for 3 minutes. Mouse anti-human CEACAM6 monoclonal antibody clone 9A6 was used at a 1:25 dilution. Sections were counterstained with Mayer's hematoxylin. Analysis of qualitative staining was focused on the type of cell stained, the pattern of staining, and its distribution. CEACAM6 staining of individual tissue samples by monoclonal antibodies was compared and graded into 4 groups from negative (-) to strongly positive $(+++)$ : (-) indicates negative staining of cytoplasm and epithelial surface; $(+)$ weakly positive staining of epithelial surface of scattered cells; $(++)$ weakly to moderately positive staining of cytoplasm and epithelial surface of most cells; and (+++) strongly positive staining of cytoplasm and epithelial surface of most cells. For all the positively stained sections, adjacent sections were also stained with monoclonal mouse $\operatorname{IgG}_{1}$ (Sigma-Aldrich) as an isotype-matched control.

Cell culture, transfection, and in vitro adhesion assays. Caco-2, T84, HT29, HCT-116, Colo205, SW480, LS174, Int-407, and HeLa cells were obtained from ATCC and cultured as described previously (47). Two oligonucleotides, 19 residues in length (5'-CCUCCUAAUAGUCAUACUA-3' specific to the human CEACAM6 mRNA and 5'-UUCUCCGAACGUGUCACGU-3' as a control), were selected for synthesis of siRNA and transfected with a cationic lipid (Lipofectamine 2000; Invitrogen) according to the manufacturer's instructions. The depletion of CEACAM6 expression by siRNA was confirmed by Western blot analysis. To determine the total number of cellassociated bacteria corresponding to adherent and intracellular bacteria, Caco-2 cells were lysed after the 3-hour infection period, and the bacteria were quantified as previously described (14).

Protein extraction and immunoblotting experiments. Cells were seeded on 6-well plates and stimulated 24 hours later with $50 \mathrm{ng} / \mathrm{ml}$ of IFN- $\gamma$ or TNF- $\alpha$ for 1 or 2 days. Medium was removed and $300 \mu$ l of lysis buffer ( $1 \%$ Triton X-100, 0.1 M NaCl, 10 mM HEPES pH 5.6, 2 mM EDTA, $4 \mathrm{mM} \mathrm{Na}_{3} \mathrm{VO}_{4}, 40 \mathrm{mM} \mathrm{NaF}$ ) supplemented with protease inhibitor cocktail (Complete Mini; Roche) was added. Lysates were harvested and passed 10 times through a $21 \mathrm{G}$ needle. The soluble protein fraction was obtained by centrifugation at $10,000 \mathrm{~g}$ for 30 minutes at $4{ }^{\circ} \mathrm{C}$. Protein concentration was determined using the DC Protein Assay kit (Bio-Rad). Ten micrograms of proteins from all cells were separated on 4\%-12\% Tris-glycine gels (Invitrogen), blotted onto PVDF

membranes, and stained using mouse anti-human CEACAM6 monoclonal antibody clone 9A6 for CEACAM6 and goat anti-human $\beta$-actin polyclonal antibody (C-11; Santa Cruz Biotechnology Inc.) for $\beta$-actin.

Expression of type 1 pili by bacteria was analyzed with whole-cell extracts. After an overnight incubation at $37^{\circ} \mathrm{C}$ in $\mathrm{LB}$ broth, bacteria at the same OD were centrifuged and resuspended in SDS-PAGE loading buffer and heated for 5 minutes at $95^{\circ} \mathrm{C}$. Protein preparations were acidified with $\mathrm{HCl}$, heated for 5 minutes at $95^{\circ} \mathrm{C}$, and separated by $12 \%$ SDS-PAGE. Western immunoblotting was performed using E. coli type 1 pili antibodies (generous gift of Maryvonne Moulin-Schouleur, Pathogénie Bactérienne, UR86, INRA, Nouzilly, France). Immunoreactants were detected using HRP-anti-rabbit IgG antibody (diluted 1:10,000), ECL reagents (Amersham), and autoradiography.

Confocal microscopy. HeLa cells, HeLa cells expressing human CEACAM6, and Caco- 2 cells were seeded on sterile glass coverslips. After 24 hours for HeLa cells, or 15 days for Caco-2 cells, cells were infected for a 3-hour period with GFP-expressing LF82 bacteria (48) at an MOI of 10. Cells were stained using mouse anti-human CEACAM 6 monoclonal antibody clone 9A6 and Texas red-conjugated anti-mouse IgG (Vector Laboratories) as secondary antibody. Cells were observed with a Zeiss LSM 510 Meta confocal microscope. A 3D reconstruction was performed using Velocity software (version 3.7.0).

Statistics. The $\chi^{2}$ test was used to compare adhesion to CD and control enterocytes and to analyze immunohistochemistry staining unless the variables required a 2-tailed Fisher's exact test. A $P$ value less than or equal to 0.05 was considered statistically significant. For the analysis of significant differences of the other data, 1 -tailed Student's $t$ test was used. A $P$ value less than or equal to 0.05 was considered statistically significant.

\section{Acknowledgments}

This study was supported by the Ministère de la Recherche et de la Technologie (EA3844), INRA (USC-2018), and by grants from the Association F. Aupetit (AFA) and Institut de Recherche des Maladies de l'Appareil Digestif (IRMAD, Laboratoire Astra France). We gratefully acknowledge Maryvonne Moulin-Schouleur (Pathogénie Bactérienne, UR86, INRA, Nouzilly, France) for E. coli type 1 pili antibodies. We thank Per Klemm (Center for Biomedical Microbiology, Lyngby, Denmark) for providing plasmid pPil38, Oliver Billker and Thomas F. Meyer (Max-Planck-Institut fur Infektionsbiologie, Berlin, Germany) for providing HeLa cells transfected with cloned human CEACAM6 cDNA, and Cecilia Berin for critical reading of the manuscript.

Received for publication September 28, 2006, and accepted in revised form March 27, 2007.

Address correspondence to: Arlette Darfeuille-Michaud, Pathogénie Bactérienne Intestinale, Laboratoire de Bactériologie, CBRV, 28 place Henri Dunant, 63001 Clermont-Ferrand, France. Phone: 33-4-73-17-79-97; Fax: 33-4-73-17-83-71; E-mail: arlette.darfeuillemichaud@u-clermont1.fr.

1. Loftus, E.V., Jr., et al. 1998. Crohn's disease in Olmsted County, Minnesota, 1940-1993: incidence, prevalence, and survival. Gastroenterology. 114:1161-1168.

2. Elson, C.O. 2000. Commensal bacteria as targets in Crohn's disease. Gastroenterology. 119:254-257.

3. Podolsky, D.K. 2002. Inflammatory bowel disease. N. Engl. J. Med. 347:417-429.

4. Shanahan, F. 2002. Crohn's disease. Lancet. 359:62-69.

5. Hugot, J.P., et al. 2001. Association of NOD2 leu- cine-rich repeat variants with susceptibility to Crohn's disease. Nature. 411:599-603.

6. Ogura, Y., et al. 2001. A frameshift mutation in NOD2 associated with susceptibility to Crohn's disease. Nature. 411:603-606.

7. Lederman, E., et al. 1997. Bacterial overgrowth in the neoterminal ileum after ileocolonic resection for Crohn's disease [abstract]. Gastroenterology. 112:A1023.

8. Neut, C., et al. 2002. Changes in the bacterial flora of the neoterminal ileum after ileocolonic resection for
Crohn's disease. Am.J. Gastroenterol. 97:939-946.

9. Darfeuille-Michaud, A., et al. 1998. Presence of adherent Escherichia coli strains in ileal mucosa of patients with Crohn's disease. Gastroenterology. 115:1405-1413.

10. Swidsinski, A., et al. 2002. Mucosal flora in inflammatory bowel disease. Gastroenterology. 122:44-54.

11. Masseret, E., et al. 2001. Genetically related Escherichia coli strains associated with Crohn's disease. Gut. 48:320-325.

12. Boudeau, J., Glasser, A.L., Masseret, E., Joly, B., and 
Darfeuille-Michaud, A. 1999. Invasive ability of an Escherichia coli strain isolated from the ileal mucosa of a patient with Crohn's disease. Infect. Immun. 67:4499-4509.

13. Glasser, A.L., et al. 2001. Adherent invasive Escherichia coli strains from patients with Crohn's disease survive and replicate within macrophages without inducing host cell death. Infect. Immun. 69:5529-5537.

14. Boudeau, J., Barnich, N., and Darfeuille-Michaud, A. 2001. Type 1 pili-mediated adherence of Escherichia coli strain LF82 isolated from Crohn's disease is involved in bacterial invasion of intestinal epithelial cells. Mol. Microbiol. 39:1272-1284.

15. Darfeuille-Michaud, A., et al. 2004. High prevalence of adherent-invasive Escherichia coli associated with ileal mucosa in Crohn's disease. Gastroenterology. 127:412-421

16. Pouttu, R., et al. 1999. Amino acid residue Ala-62 in the FimH fimbrial adhesin is critical for the adhesiveness of meningitis-associated Escherichia coli to collagens. Mol. Microbiol. 31:1747-1757.

17. Kukkonen, M., et al. 1993. Basement membrane carbohydrate as a target for bacterial adhesion: binding of type I fimbriae of Salmonella enterica and Escherichia coli to laminin. Mol. Microbiol. 7:229-237.

18. Pak, J., Pu, Y., Zhang, Z.T., Hasty, D.L., and Wu, X.R. 2001. Tamm-Horsfall protein binds to type 1 fimbriated Escherichia coli and prevents E. coli from binding to uroplakin Ia and Ib receptors. J. Biol. Chem. 276:9924-9930.

19. Sauter, S.L., Rutherfurd, S.M., Wagener, C., Shively, J.E., and Hefta, S.A. 1993. Identification of the specific oligosaccharide sites recognized by type 1 fimbriae from Escherichia coli on nonspecific crossreacting antigen, a CD66 cluster granulocyte glycoprotein. J. Biol. Chem. 268:15510-15516.

20. Leusch, H.G., et al. 1990. Escherichia coli of human origin binds to carcinoembryonic antigen (CEA) and non-specific crossreacting antigen (NCA). FEBS Lett. 261:405-409.

21. Burtin, P., Quan, P.C., and Sabine, M.C. 1975. Nonspecific cross reacting antigen as a marker for human polymorphs, macrophages and monocytes. Nature. 255:714-716

22. Darfeuille-Michaud, A., et al. 1990. Adhesion of enterotoxigenic Escherichia coli to the human colon carcinoma cell line Caco-2 in culture. Infect. Immun. 58:893-902.

23. Oro, H.S., Kolsto, A.B., Wenneras, C., and Svennerholm, A.M. 1990. Identification of asialo GM1 as a binding structure for Escherichia coli colonization factor antigens. FEMS Microbiol. Lett. 60:289-292.

24. Knutton, S., Lloyd, D.R., Candy, D.C., and McNeish,
A.S. 1985. Adhesion of enterotoxigenic Escherichia coli to human small intestinal enterocytes. Infect. Immun. 48:824-831.

25. Kenny, B., et al. 1997. Enteropathogenic E. coli (EPEC) transfers its receptor for intimate adherence into mammalian cells. Cell. 91:511-520.

26. DeVinney, R., et al. 1999. Enterohemorrhagic Escherichia coli $\mathrm{O} 157: \mathrm{H} 7$ produces Tir, which is translocated to the host cell membrane but is not tyrosine phosphorylated. Infect. Immun. 67:2389-2398.

27. Servin, A.L. 2005. Pathogenesis of Afa/Dr diffusely adhering Escherichia coli. Clin. Microbiol. Rev. 18:264-292.

28. Sokurenko, E.V., Courtney, H.S., Maslow, J., Siitonen, A., and Hasty, D.L. 1995. Quantitative differences in adhesiveness of type 1 fimbriated Escherichia coli due to structural differences in fim H genes. J. Bacteriol. 177:3680-3686.

29. Rhodes, J.M., and Campbell, B.J. 2002. Inflammation and colorectal cancer: IBD-associated and sporadic cancer compared. Trends Mol. Med. 8:10-16.

30. Rhodes, J.M. 1996. Unifying hypothesis for inflammatory bowel disease and associated colon cancer: sticking the pieces together with sugar. Lancet. 347:40-44.

31. Tysk, C., et al. 1991. Colonic glycoproteins in monozygotic twins with inflammatory bowel disease. Gastroenterology. 100:419-423.

32. Grunert, F., Daniel, S., Nagel, G., von Kleist, S., and Jantscheff, P. 1995. CD66b, CD66c and carcinoembryonic antigen (CEA) are independently regulated markers in sera of tumor patients. Int. J. Cancer. 63:349-355.

33. Neumaier, M., Paululat, S., Chan, A., Matthaes, P., and Wagener, C. 1993. Biliary glycoprotein, a potential human cell adhesion molecule, is downregulated in colorectal carcinomas. Proc. Natl. Acad. Sci. U. S. A. 90:10744-10748

34. Jantscheff, P., et al. 2003. Expression of CEACAM6 in resectable colorectal cancer: a factor of independent prognostic significance. J. Clin. Oncol. 21:3638-3646.

35. Scholzel, S., et al. 2000. Carcinoembryonic antigen family members CEACAM6 and CEACAM7 are differentially expressed in normal tissues and oppositely deregulated in hyperplastic colorectal polyps and early adenomas. Am. J. Pathol. 156:595-605.

36. Caccamo, D., Telenta, M., and Celener, D. 1989. Concanavalin A binding sites in fetal, adult, transitional, and malignant rectosigmoid mucosa. Hum. Pathol. 20:1186-1192.

37. Fahlgren, A., et al. 2003. Interferon-gamma tempers the expression of carcinoembryonic antigen family molecules in human colon cells: a possible role in innate mucosal defence. Scand. J. Immunol.
58:628-641.

38. Muenzner, P., Naumann, M., Meyer, T.F., and Gray-Owen, S.D. 2001. Pathogenic Neisseria trigger expression of their carcinoembryonic antigenrelated cellular adhesion molecule 1 (CEACAM1; previously CD66a) receptor on primary endothelial cells by activating the immediate early response transcription factor, nuclear factor-kappaB. J. Biol. Chem. 276:24331-24340.

39. Betis, F., et al. 2003. Afa/Dr diffusely adhering $E s c h$ erichia coli infection in T84 cell monolayers induces increased neutrophil transepithelial migration, which in turn promotes cytokine-dependent upregulation of decay-accelerating factor (CD55), the receptor for $\mathrm{Afa} / \mathrm{Dr}$ adhesins. Infect. Immun. 71:1774-1783.

40. Avadhanula, V., Rodriguez, C.A., Ulett, G.C., Bakaletz, L.O., and Adderson, E.E. 2006. Nontypeable Haemophilus influenzae adheres to intercellular adhesion molecule 1 (ICAM-1) on respiratory epithelial cells and upregulates ICAM-1 expression. Infect. Immun. 74:830-838.

41. Reimund, J.M., et al. 1996. Increased production of tumour necrosis factor-alpha interleukin-1 beta, and interleukin- 6 by morphologically normal intestinal biopsies from patients with Crohn's disease. Gut. 39:684-689.

42. Chamaillard, M., Iacob, R., Desreumaux, P., and Colombel, J.F. 2006. Advances and perspectives in the genetics of inflammatory bowel diseases. Clin. Gastroenterol. Hepatol. 4:143-151.

43. Gower-Rousseau, C., et al. 1994. Incidence of inflammatory bowel disease in northern France (1988-1990). Gut. 35:1433-1438.

44. Datsenko, K.A., and Wanner, B.L. 2000. One-step inactivation of chromosomal genes in Escherichia coli K-12 using PCR products. Proc. Natl. Acad. Sci.U. S. A. 97:6640-6645.

45. Chaveroche, M.K., Ghigo, J.M., and d'Enfert, C. 2000. A rapid method for efficient gene replacement in the filamentous fungus Aspergillus nidulans. Nucleic Acids Res. 28:E97.

46. Klemm, P., Jorgensen, B.J., van Die, I., de Ree, H., and Bergmans, H. 1985. The fim genes responsible for synthesis of type 1 fimbriae in Escherichia coli, cloning and genetic organization. Mol. Gen. Genet. 199:410-414.

47. Hisamatsu, T., et al. 2003. CARD15/NOD2 functions as an antibacterial factor in human intestinal epithelial cells. Gastroenterology. 124:993-1000.

48. Bringer, M.A., Glasser, A.L., Tung, C.H., Meresse, S., and Darfeuille-Michaud, A. 2006. The Crohn's disease-associated adherent-invasive Escherichia coli strain LF82 replicates in mature phagolysosomes within J774 macrophages. Cell. Microbiol. 8:471-484. 